\title{
Article
}

\section{Probing Charge Carrier Dynamics to Unveil the Role of Surface Ligands in HgTe Narrow Band Gap Nanocrystals}

Bertille Martinez, Clément Livache, Nicolas Goubet, Amardeep Jagtap, Hervé Cruguel, Abdelkarim Ouerghi, Emmanuelle Lacaze, Mathieu G. Silly, and Emmanuel Lhuillier

J. Phys. Chem. C, Just Accepted Manuscript • DOI: 10.1021/acs.jpcc.7b09972 • Publication Date (Web): 14 Dec 2017

Downloaded from http://pubs.acs.org on December 15, 2017

\section{Just Accepted}

"Just Accepted" manuscripts have been peer-reviewed and accepted for publication. They are posted online prior to technical editing, formatting for publication and author proofing. The American Chemical Society provides "Just Accepted" as a free service to the research community to expedite the dissemination of scientific material as soon as possible after acceptance. "Just Accepted" manuscripts appear in full in PDF format accompanied by an HTML abstract. "Just Accepted" manuscripts have been fully peer reviewed, but should not be considered the official version of record. They are accessible to all readers and citable by the Digital Object Identifier (DOI®). "Just Accepted" is an optional service offered to authors. Therefore, the "Just Accepted" Web site may not include all articles that will be published in the journal. After a manuscript is technically edited and formatted, it will be removed from the "Just Accepted" Web site and published as an ASAP article. Note that technical editing may introduce minor changes to the manuscript text and/or graphics which could affect content, and all legal disclaimers and ethical guidelines that apply to the journal pertain. ACS cannot be held responsible for errors or consequences arising from the use of information contained in these "Just Accepted" manuscripts. 


\title{
Probing Charge Carrier Dynamics to Unveil the Role of Surface Ligands in HgTe Narrow Band Gap Nanocrystals
}

\author{
Bertille Martinez ${ }^{1}$, Clément Livache ${ }^{1}$, Nicolas Goubet ${ }^{1}$, Amardeep Jagtap ${ }^{1}$, Hervé Cruguel $^{1}$, Abdelkarim \\ Ouerghi ${ }^{2}$, Emmanuelle Lacaze ${ }^{1}$, Mathieu G. Silly ${ }^{3}$, Emmanuel Lhuillier $^{1^{*}}$ \\ ${ }^{1}$ Sorbonne Universités, UPMC Univ. Paris 06, CNRS-UMR 7588, Institut des NanoSciences de Paris, 4 place \\ Jussieu, 75005 Paris, France \\ ${ }^{2}$ Centre de Nanosciences et de Nanotechnologies, CNRS, Univ. Paris-Sud, Université Paris-Saclay, C2N - \\ Marcoussis, 91460 Marcoussis, France \\ ${ }^{3}$ Synchrotron-SOLEIL, Saint-Aubin, BP48, F91192 Gif sur Yvette Cedex, France
}

\begin{abstract}
Colloidal nanocrystals are an interesting platform for the design of low cost optoelectronic devices especially in the infrared range of wavelengths. Mercury chalcogenides have reached high maturity to address wavelengths above the telecom range $(1.5 \mu \mathrm{m})$. However, no screening of the surface chemistry influence has been conducted yet. In this paper, we systematically probe the influence of a series of ligands: $\mathrm{Cl}^{-}, \mathrm{SCN}^{-}, 1,2$ ethanedithiol, 1,4 benzenedithiol, 1 octanethiol, 1 butanethiol, $\mathrm{As}_{2} \mathrm{~S}_{3}, \mathrm{~S}^{2-}$ on the photoconductive properties of HgTe nanocrystal thin films. A high bandwidth, large dynamic transient photocurrent setup is used to determine the photocarrier dynamics. Two regimes are clearly identified. At early stage (few ns) a fast decay of the photocurrent is resulting from recombination and trapping. Then transport enters in a multiple trapping regime where carriers present a continuously decreasing effective value of their mobility. The power law dependence of the conductance can be used to estimate the trap carrier density and determine the value of the Urbach energy (35 to $50 \mathrm{meV}$ ). We demonstrate that a proper choice of ligand is necessary for a trade-off between the material performance $(\mu \tau$ product) and the quality of the surface passivation (to keep a low Urbach energy).
\end{abstract}

To whom correspondence should be sent: el@insp.upmc.fr 


\section{INTRODUCTION}

Colloidal quantum dots (CQD) are promising candidates for the emergence of low cost optoelectronic devices ${ }^{1}$. This is especially true in the infrared range of wavelengths where current technologies remain driven by epitaxially grown semiconductors of II-VI ( $\mathrm{HgCdTe}$ ) and III-V materials. CQD by combining (i) the lack of constrain of an epitaxial growth, (ii) an ease of processing similar to organic electronics and (iii) a strong tunability of the optical absorption from $\mathrm{UV}$ to $\mathrm{THz}^{2}$ offer a unique platform to revisit infrared optoelectronics ${ }^{3}$.

To reach wavelengths in the infrared range and especially in the short wavelength infrared range (SWIR) and mid wave infrared range $\left(\mathrm{MWIR}^{4}\right)$, narrow band gap materials have to be used ${ }^{5}$. Among potential candidates, mercury chalcogenides $\left(\mathrm{HgS}^{6,7}, \mathrm{HgSe}^{8-10}\right.$ and $\mathrm{HgTe}^{11}$ ) are the most mature materials, combining a tunable infrared absorption ${ }^{12,13}$ with photoconductive ${ }^{14}$ properties. In spite of huge progresses reported over the past decades ${ }^{15}$, which include the report of photovoltaic devices ${ }^{16}$, intraband transition based photodetector ${ }^{1,17}$, plasmonic devices $^{18,19}$ and multicolor devices ${ }^{20,21}$, no rational design of the material has been conducted. As a result, very little is known ${ }^{22}$ about the electronic structure of these narrow band gap confined CQD. Particularly, no screening of the surface chemistry influence has been reported yet in contrast to what has been reported for PbS CQD ${ }^{23}$ for example.

In a photoconductor, the product of the mobility $(\mu)$ by the lifetime $(\tau)$ is driving the value of the photocurrent magnitude ${ }^{24}$. While DC photocurrent measurements give access to the product $\mu \cdot \tau$, accessing separately to these two parameters is more challenging. To do so, we use a combination of field effect transistor measurements and transient photocurrent measurements to extract respectively the mobility and carrier lifetime. Our time resolved measurements, conducted over 6 orders of magnitude of duration, exhibit two different regimes. At short time scale the carrier population quickly thermalizes and is followed by a long tail power law behavior, which results from a multi trapping transport process. From the latter, we are able to extract the Urbach energy which describes the trap state distribution. Finally using X-ray photoemission we are able to propose a full electronic spectrum of the quantum and trap states in absolute energy scale.

\section{METHODS}

\section{Chemicals}

Mercury chloride $\left(\mathrm{HgCl}_{2}\right.$, Strem Chemicals, 99\%), tellurium powder (Te, Sigma-Aldrich, 99.99\%), trioctylphosphine (TOP, Cytek, 90\%), oleylamine (Acros, 80-90\%), 1,octanethiol (OSH, Fluka), 1,butanthiol (BuSH, Sigma Aldrich), benzedithiol (BeSH, Alfa), dodecanethiol (DDT, Sigma-Aldrich, 98\%), sodium sulfide nonahydrate $\left(\mathrm{Na}_{2} \mathrm{~S}, 9 \mathrm{H}_{2} \mathrm{O}\right.$, Sigma Aldrich, 98.0\%), arsenic trisulfide $\left(\mathrm{As}_{2} \mathrm{~S}_{3}\right.$, Alfa Aesar, 99.9\%), 1,2,ethanedithiol (EDT, Fluka, 98.0\%), ammonium chloride $\left(\mathrm{NH}_{4} \mathrm{Cl}\right.$, Sigma Aldrich), ammonium thiocyanate $\left(\mathrm{NH}_{4} \mathrm{SCN}\right.$, Sigma Aldrich), butylamine (Sigma Aldrich), hydrochloric acid $\left(\mathrm{HCl}\right.$, Mieuxa, 25\%), lithium perchlorate $\left(\mathrm{LiClO}_{4}\right.$, Sigma-Aldrich, $\left.98 \%\right)$, polyethylene glycol $\left(\mathrm{PEG}, \mathrm{M}_{\mathrm{w}}\right.$ $=6 \mathrm{~kg}^{\mathrm{mol}}{ }^{-1}$ ), chloroform (Carlo Erba), ethanol absolute anhydrous (Carlo Erba, 99.9\%), methanol (Carlo Erba, 99.8\%), acetone (Carlo Erba, 99.8\%), n-hexane (Carlo Erba), n-octane (SDS, 99\%), toluene (Carlo Erba, 99.3\%), Nmethylformamide (NMF, VWR, 98\%). All chemical are used as received, except oleylamine which is centrifuged before used. Mercury compounds are highly toxic. Handle them with special care.

\section{Material synthesis}

$1 \mathrm{M}$ TOPTe precursor: $2.54 \mathrm{~g}$ of Te powder are mixed in $20 \mathrm{~mL}$ of TOP in a three neck flask. The flask is put under vacuum at room temperature for $5 \mathrm{~min}$ and then the temperature is raised at $100^{\circ} \mathrm{C}$ and the degassing is further conducted over the next 20 minutes. The atmosphere is then switched to $\mathrm{Ar}$ and the temperature raised to $275^{\circ} \mathrm{C}$. The solution is stirred until a clear orange solution is obtained. The flask is then cooled down and the color switches 
to yellow. At room temperature, the flask is degassed again for $10 \mathrm{~min}$. Finally, this solution is transferred to an air free glove box for storage.

HgTe synthesis: In a $25 \mathrm{~mL}$ three neck flask, $135 \mathrm{mg}$ of $\mathrm{HgCl}_{2}$ and $15 \mathrm{~mL}$ of oleylamine are degassed at $110^{\circ} \mathrm{C}$ for $1 \mathrm{~h}$. Under $\mathrm{Ar}$ at $80^{\circ} \mathrm{C}$, a solution containing $0.5 \mathrm{~mL}$ of $1 \mathrm{M}$ TOPTe and $4.5 \mathrm{~mL}$ of oleylamine is quickly injected. The reaction is conducted for 3 minutes. The reaction is quenched by a mixture of chloroform (15 mL), DDT (1.5 mL) and TOP (several drops) and the flask is cooled down using fresh air. The particles are precipitated in ethanol and dispersed in toluene or 9:1 hexane-octane depending on the ligand-exchange procedure.

\section{Ligand exchange procedures}

On film procedure: Solution of HgTe nanoparticles in 9:1 hexane-octane is dropcasted on a clean substrate. Once the solvent is completely evaporated, ligand exchange is performed on the sample to remove DDT and replace it by $\mathrm{OSH}, \mathrm{BuSH}, \mathrm{BeSH}, \mathrm{EDT}, \mathrm{S}^{2-}, \mathrm{Cl}^{-}, \mathrm{SCN}^{-}$or $\mathrm{As}_{2} \mathrm{~S}_{3}$. These ligands are simply dissolved in ethanol to reach a $1 \mathrm{wt} \%$ solution, except for $\mathrm{As}_{2} \mathrm{~S}_{3}$ which is first dissolved in butylamine before a few drops of this solution are mixed to about $20 \mathrm{~mL}$ of ethanol. To perform ligand exchange, the film is dipped into the solution containing the new ligands for $90 \mathrm{~s}$. Then the film is rinsed with ethanol for $30 \mathrm{~s}$. To get a thicker film (around $100 \mathrm{~nm}$ at the end), this step can be repeated 3 to 5 times.

Liquid phase procedure: $0.5 \mathrm{~mL}$ of a concentrated solution of $\mathrm{Na}_{2} \mathrm{~S}$ in NMF is poured into a solution of $\mathrm{HgTe}$ nanoparticles in toluene (about $2 \mathrm{~mL}$ ). $1 \mathrm{~mL}$ of hexane is added to help phase dissociation and the mixture is sonicated. The particles migrate from the apolar phase (toluene+hexane) down to the polar phase (NMF) by exchanging their ligands. The upper phase is removed and the NMF phase is washed 3 times with hexane. Then, $2 \mathrm{~mL}$ of ethanol and $2 \mathrm{~mL}$ of toluene are poured into the NMF solution to destabilize the particles and help them flocculating. The solution is then centrifuged at $3300 \mathrm{rpm}$ for $3 \mathrm{~min}$ and the particles are dispersed in fresh NMF (a few milliliters). This liquid phase ligand exchange procedure can also be done with $\mathrm{As}_{2} \mathrm{~S}_{3}$, the $\mathrm{Na}_{2} \mathrm{~S}$ in NMF solution is then replaced by $0.5 \mathrm{~mL}$ of a saturated solution of $\mathrm{As}_{2} \mathrm{~S}_{3}$ in butylamine, diluted in $0.5 \mathrm{~mL}$ of NMF. In this case the films are obtained by dropcasting the solution onto the electrodes on a hot plate $\left(100^{\circ} \mathrm{C}\right)$.

\section{Electrodes fabrication}

$\mathrm{Si} / \mathrm{SiO}_{2}$ substrate for electrodes: The surface of a $\mathrm{Si} / \mathrm{SiO}_{2}$ wafer ( $400 \mathrm{~nm}$ oxide layer) is cleaned by sonication in acetone. The wafer is rinsed with isopropanol and finally cleaned using an $\mathrm{O}_{2}$ plasma. AZ 5214E resist is spin-coated and baked at $110^{\circ} \mathrm{C}$ for $90 \mathrm{~s}$. The substrate is exposed under UV through a pattern mask for $2 \mathrm{~s}$. The film is further baked at $125^{\circ} \mathrm{C}$ for $2 \mathrm{~min}$ to invert the resist. Then a $40 \mathrm{~s}$ flood exposure is performed. The resist is developed using a bath of AZ 326 for $32 \mathrm{~s}$, before being rinsed in pure water. We then deposit a $5 \mathrm{~nm}$ chromium layer and $80 \mathrm{~nm}$ gold layer using a thermal evaporator. The lift-off is performed by dipping the film in acetone for $1 \mathrm{~h}$. The electrodes are finally rinsed using isopropanol and dried by an air flow. The electrodes are $2.5 \mathrm{~mm}$ long and spaced by $20 \mu \mathrm{m}$. These electrodes are used for DC measurements (IV curves and transistor measurements).

PET/ITO electrodes: The surface of a PET/ITO wafer is cleaned by rinsing with acetone and isopropanol. AZ 5214E resist is spin-coated and baked at $110^{\circ} \mathrm{C}$ for $90 \mathrm{~s}$. The substrate is exposed under UV through a pattern mask for $4 \mathrm{~s}$. The resist is developed using a bath of AZ 326 for $45 \mathrm{~s}$, before being rinsed in pure water. We then proceed to the etching of the electrodes by dipping the substrate in $25 \% \mathrm{HCl}$ for $10 \mathrm{~s}$ and rince with pure water to quench. The electrodes are finally rinsed using isopropanol and dried by an air flow. The electrodes are $1 \mathrm{~mm}$ long and spaced by $20 \mu \mathrm{m}$. These electrodes are used for transient photocurrent measurements. Transparent electrodes are used to avoid any heating effect of the photoresponse which tends to introduce drift.

Electrolyte gating: For electrolyte gating we first mix in a glove box $0.5 \mathrm{~g}$ of $\mathrm{LiClO}_{4}$ with $2.3 \mathrm{~g}$ of $\mathrm{PEG}\left(\mathrm{M}_{\mathrm{W}}=6 \mathrm{~kg} \cdot \mathrm{mol}^{-}\right.$ $\left.{ }^{1}\right)$. The vial is heated at $170^{\circ} \mathrm{C}$ on a hot plate for $2 \mathrm{~h}$ until the solution gets clear. To use the electrolyte, the solution is warmed around $100^{\circ} \mathrm{C}$ and brushed on the top of the HgTe film. 


\section{Electrical measurements}

DC transport: The sample is connected to a Keithley $2634 \mathrm{~b}$ which controls the drain bias $\left(\mathrm{V}_{\mathrm{DS}}\right)$ between -2 and $+2 \mathrm{~V}$ with a step of $10 \mathrm{mV}$, and measures the associated current $\left(\mathrm{I}_{\mathrm{DS}}\right)$. This measurement is made in the dark and under illumination (green diode, $532 \mathrm{~nm}, 25 \mathrm{~mW}$ ).

Transistor measurements: The sample is connected to a Keithley 2634b which sets the drain bias $\left(\mathrm{V}_{\mathrm{DS}}=0.01 \mathrm{~V}\right)$, controls the gate bias $\left(\mathrm{V}_{\mathrm{GS}}\right)$ between -2 and $+2 \mathrm{~V}$ with a step of $1 \mathrm{mV}$ and measures the associated currents $\mathrm{I}_{\mathrm{DS}}$ and $\mathrm{I}_{\mathrm{GS}}$. The measurements are repeated 5 times to ensure the obtained curves are stable.

Time - resolved measurements: For the time-resolved measurements, we use a pulsed laser (Crylas FTSS 355-50) at $355 \mathrm{~nm}$. The laser beam is around $1 \mathrm{~mm}$ in diameter (ie poorly focused) to prevent excessive heating of the sample, which may lead to a drift of the photoresponse. The pulses are $1 \mathrm{~ns}$ long and repeated every $10 \mathrm{~ms}(100 \mathrm{~Hz})$. A photodiode is used to trigger the signal. The sample is placed in a vacuum chamber and connected as shown in Figure 3a. The bias, $50 \mathrm{~V}$, is applied by a Keithley $2432 \mathrm{~b}$. An oscilloscope (Rohde \& Schwarz, RTE 1102) acquires the outcoming signal through a $50 \Omega$ resistor, which is proportional to IDS. The response of the film is measured at different time scales, normalized, and plot in a log-log plot as shown on Figure $3 \mathrm{~b}$.

\section{DISCUSSION}

We first synthetize DDT capped HgTe CQD with an excitonic feature at $4000 \mathrm{~cm}^{-1}(\approx 0.48 \mathrm{eV}$ of band gap or $2.5 \mu \mathrm{m}$, see Figure 1a), using previously reported procedure ${ }^{25}$. The absorption cut off wavelength of this material is well suited for the detection of short wave infrared wavelengths, which is motivated by the possibility to conduct infrared imaging thanks to night glows. The material presents a tetrapodic shape, see transmission electron image in Figure $1 \mathrm{~b}$ and has a zinc blende structure $\mathrm{e}^{25}$.

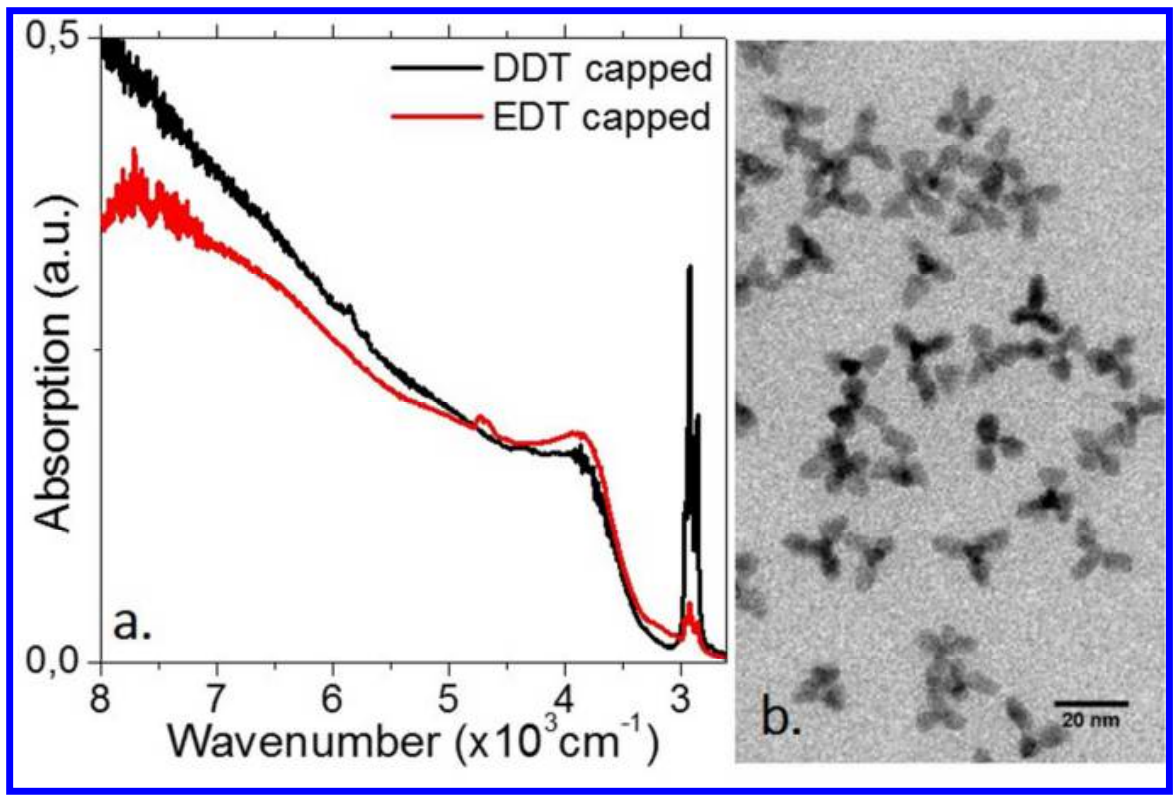

Figure 1 a. Infrared spectrum of HgTe CQD capped with dodecanethiol (DDT) and ethanedithiol (EDT). b. TEM image of the used HgTe CQD with a tetrapodic shape.

To make the material suitable for the design of infrared detectors, it is critical to exchange the initial long and insulating capping ligands (dodecanethiol) for shorter molecules, which will ensure a better interdot electronic coupling and an increase of the conductivity. We have selected a series of short ligands, which have been tested on 
nanocrystals to improve the charge transport and phototransport properties of films: $\mathrm{Cl}^{-26}, \mathrm{SCN}^{-27}$, ethanedithiol $(E D T)^{28}, \mathrm{~S}^{2-29}, \mathrm{As}_{2} \mathrm{~S}_{3}{ }^{28,30}$. In addition we tested a series of thiol ligands with different chain length to address the effect of the inter CQD distance on the photoresponse, these ligands are octanethiol (OSH), butanethiol (BuSH) and benzendithiol (BeSH). For OSH, BuSH, BeSH, $\mathrm{Cl}^{-}, \mathrm{SCN}^{-}$and $\mathrm{EDT}$, the ligand exchange is conducted on film by dipping the film in a solution of short ligands in excess. For $\mathrm{S}^{2-}$ and $\mathrm{As}_{2} \mathrm{~S}_{3}$ we have tested both on film and in solution (ie phase transfer ${ }^{24}$ ) method. The typical signature of this exchange is a significant reduction of absorption relative to the $\mathrm{C}-\mathrm{H}$ bond at $3000 \mathrm{~cm}^{-1}$, see Figure $1 \mathrm{a}$. This reduction of the amount of $\mathrm{C}-\mathrm{H}$ in the film is around $80 \%$ in the case of on film ligand exchange and it is complete for the in-solution process. Another important feature of this spectrum is the retaining of quantum confinement in nanocrystals even after ligand exchange.

The film of nanoparticles is then deposited on gold interdigitated electrodes and we follow the dark and under illumination conductance, see Figure 2a. The films of HgTe CQD present an ohmic conduction in the range of applied electric field $\left(F \approx 1 \mathrm{kV} \cdot \mathrm{cm}^{-1}\right)$. Under illumination we observe a positive photoresponse. Figure $2 \mathrm{~b}$ shows the modulation of the ratio of the conductance under illumination over conduction in the dark. This ratio typically follows a similar trend as detectivity. The general trend is the following: $\mathrm{Cl}^{-}<\mathrm{BuSH} \approx \mathrm{OSH}<\mathrm{SCN}<\mathrm{EDT}<\mathrm{As}_{2} \mathrm{~S}_{3}<\mathrm{S}^{2-}<\mathrm{BeSH}^{2}$ it appears that ligands binding through the sulfur are more suitable for photoresponse. As expected long thiol chain leads to a lower level of performance than short dithiol (EDT and BeSH) molecules. We also observe that liquid phase ligand exchange tends to increase the dark conductance leading to a smaller light/dark ratio. This effect is partly due to the necessary annealing $\left(a t 100^{\circ} \mathrm{C}\right.$ ) of the nanocrystal film to remove the polar solvent which consequently increases the dot size.

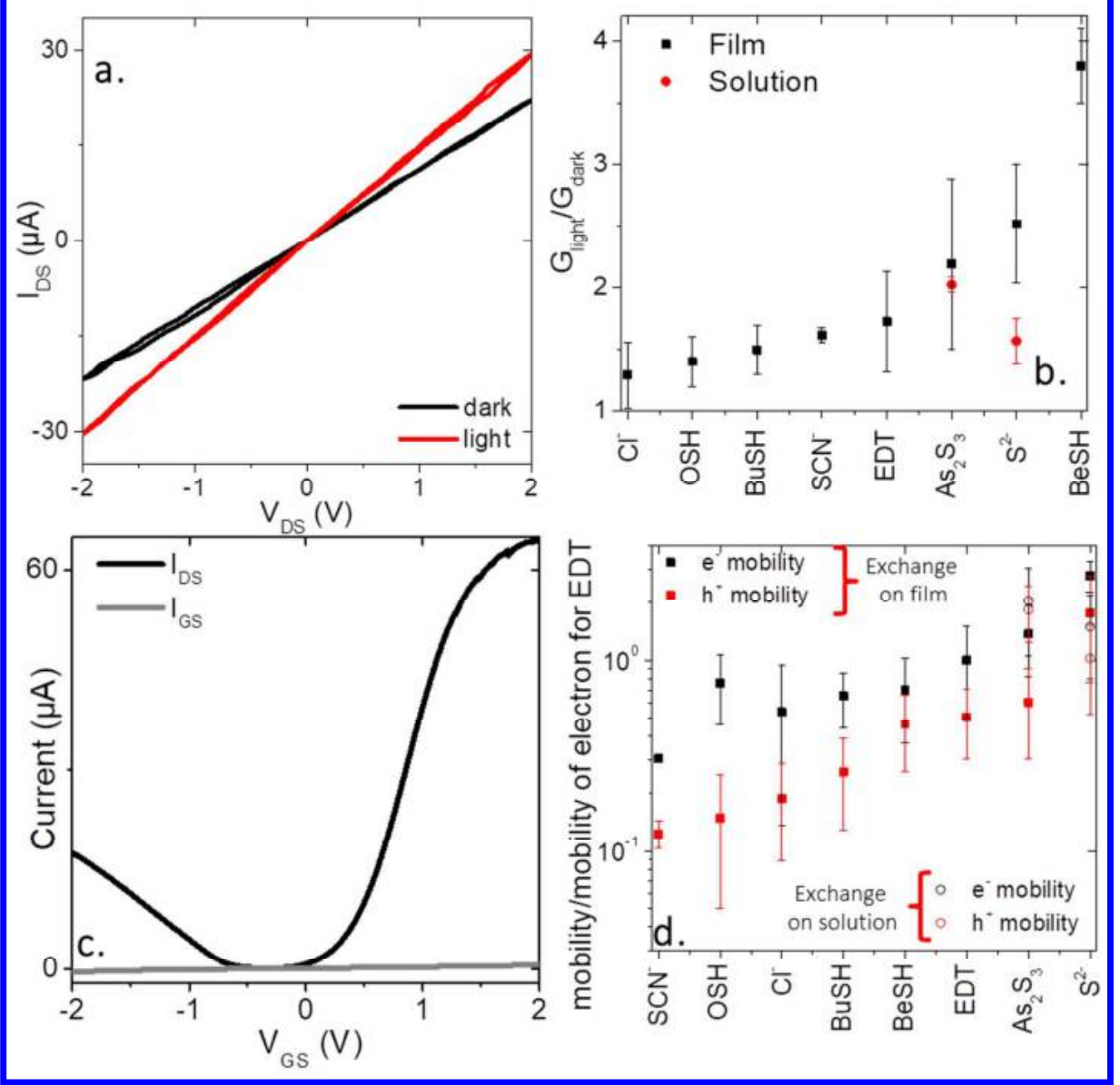

Figure 2 a. IV curves of an HgTe CQD film capped with EDT under dark condition in black and under illumination $(\lambda=532 \mathrm{~nm}-P=25 \mathrm{~mW})$ in red. $b$. Ratio of the conductance under illumination over conductance in the dark for HgTe CQD film capped with different ligands. c. Transfer curve (drain current as a function of gate bias) of an HgTe CQD film capped with EDT ligands and used as a channel of an ion gel gated transistor $\left(V_{D S}=10 \mathrm{mV}\right)$. d. Mobilities of electrons and holes for HgTe CQD films capped with different ligands normalized by the electronic mobility of EDT 
capped HgTe CQD. Error bars have been determined by repeating the measurement for each samples at least three times.

The photocurrent magnitude is driven by the product of the mobility by the time, and this product can be easily obtained with DC measurements. To go further in the understanding of these materials, we want to determine the contribution of each factor and so, to measure independently these two quantities. The mobility of thin films is easy to access through field effect transistor measurements ${ }^{3}$. To do so, we build an ion gel gated transistor, where the film of HgTe CQD is connected to a quasi-solid electrolytic gate ${ }^{31}$. The latter combines several advantages: (i) it is air operable, (ii) it presents a large gate capacitance, which allows tuning of carrier density up to several carriers per nanocrystal and finally (iii) it is possible to gate thick films, which is critical with in mind the design of strongly absorbing films for photodetection.

HgTe CQD films present an ambipolar behavior where both holes (under negative gate bias) and electrons (under positive gate bias) can contribute to the transport, see Figure $2 \mathrm{c}$. This behavior is the result of the narrow band gap nature of the HgTe CQD with respect to the ability of the electrolyte gating to tune the Fermi level over several hundred meV. We observe that the charge neutrality point (ie the bias at which the conduction switches from hole to electron) is systematically at negative bias. This suggests that the initial HgTe CQD are n-doped which will be further discussed in the last part of the article. The derivative of the transfer curve is directly proportional to the carrier mobility, however electrolyte gating is not well suited to extract absolute value of the mobility because the gate capacitance is not accurately known. Nevertheless we can compare the relative mobility obtained for the different capping ligands, see Figure $2 \mathrm{~d}$. We systematically observe that the electron mobility is a factor two to three times higher than the hole mobility. Moreover the general trends in term of mobility is quite close to the one observed for photocurrent $\mathrm{SCN}^{-}<\mathrm{OSH}<\mathrm{Cl}^{-}<\mathrm{BuSH}<\mathrm{BeSH}<\mathrm{EDT}<\mathrm{As}_{2} \mathrm{~S}_{3}<\mathrm{S}^{2-}$.

To extract the photocarrier lifetime, we build a high bandwidth $(\mathrm{GHz})$ transient photocurrent setup, see Figure 3a for a scheme of the setup. It is common to estimate such lifetime from the time resolved photoluminescence (PL); however this is difficult to conduct on narrow band gap materials for which the PL efficiency is intrinsically low. Moreover, the PL measurement is conducted in very different configuration (ie generally in solution or on film without applied electric field) which may introduce significant deviations. The specificity of our setup comes from its ability to probe the dynamics on a broad range of duration, typically from ns to ms. From this configuration, we are able to measure the dynamics on the film under an applied electric field, in order to probe transport dynamics. As a result not only are we able to probe the initial carrier density relaxation but also longer processes including trapping and detrapping. A typical curve of conductance as a function of time is shown in Figure $3 \mathrm{~b}$. The curve can be splitted in three main parts. At extremely early time $(<n s)$ we observe a rise of the conductance, which duration follows the laser pulse duration. Then, we observe a fast decay (in the 1 to $10 \mathrm{~ns}$ range), this regime can be fitted with a single exponential and the decay time is typically a few ns, see Figure 3c. This time scale can be compared to the hopping time $\tau_{h o p}$, the time for a carrier to get transported from one CQD to the next one. From the Einstein relationship we can evaluate the hopping time: $\tau_{\text {hop }}=\frac{2 e R^{2}}{3 \mu k_{b} T}$, with $e$ the proton charge, $R$ the nanoparticle radius, $\mu$ the mobility, $k_{b}$ the Boltzmann constant and $T$ the temperature $(300 \mathrm{~K})$. Assuming a mobility of $10^{-3} \mathrm{~cm}^{2} \mathrm{~V}^{-1} \mathrm{~s}^{-1}$ from previous report, ${ }^{27}$ we estimate the hopping time to be $\approx 3 \mathrm{~ns}$. Since the early decay that is observed occurs at a time scale shorter than the hopping time, it results from a change of carrier density at the nanocrystal scale rather than a change of number of carriers due to transport from one nanocrystal to another. Gao et al in a recent investigation of the photocurrent dynamics at very short time scale ( $p$ s to few $n s$ ) arrived at the same conclusion ${ }^{32,33}$. Thus the observed decay rate is the combination of two processes: $(i)$ an interband bimolecular recombination of photogenerated electron hole pair with a decay rate $k_{\text {inter }}^{r e c}$ and (ii) a carrier trapping with dynamics described by $k_{\text {traps }}^{\text {rec }}$. From this lifetime, we are able to estimate the mobility lifetime product. To take into account both the electron and hole mobilities, we actually evaluate the quantity $\sqrt{\mu_{e} \cdot \mu_{h}} \cdot \tau$, see Figure 3 c. The obtained ordering of the ligand is actually very well correlated with the photoresponsivity measurement, with clearly lower performance ACS Paragon Plus Environment 
from long thiols ( $\mathrm{OSH}, \mathrm{BuSH}), \mathrm{SCN}^{-}$and $\mathrm{Cl}^{-}$, intermediate performance for EDT and $\mathrm{BeSH}$ and higher performances for the two exchanges which are $\mathrm{S}^{2-}$ on film and $\mathrm{As}_{2} \mathrm{~S}_{3}$ in solution. Overall a good agreement is obtained between photoconductive signal $G_{\text {light }} / G_{\text {dark }}$ and the $\sqrt{\mu_{e} \cdot \mu_{h}} \cdot \tau$ product, except in the case of BeSH which photoresponse is better than predicted by the mobility lifetime product. This might result from a decrease of the dark current which can be associated to a shift of the band with respect to the Fermi level. The latter leads to a change of doping and to the dark current value, as recently highlighted in the case of $\mathrm{PbS}^{23}$ and $\mathrm{HgSe}^{34}$ CQDs.

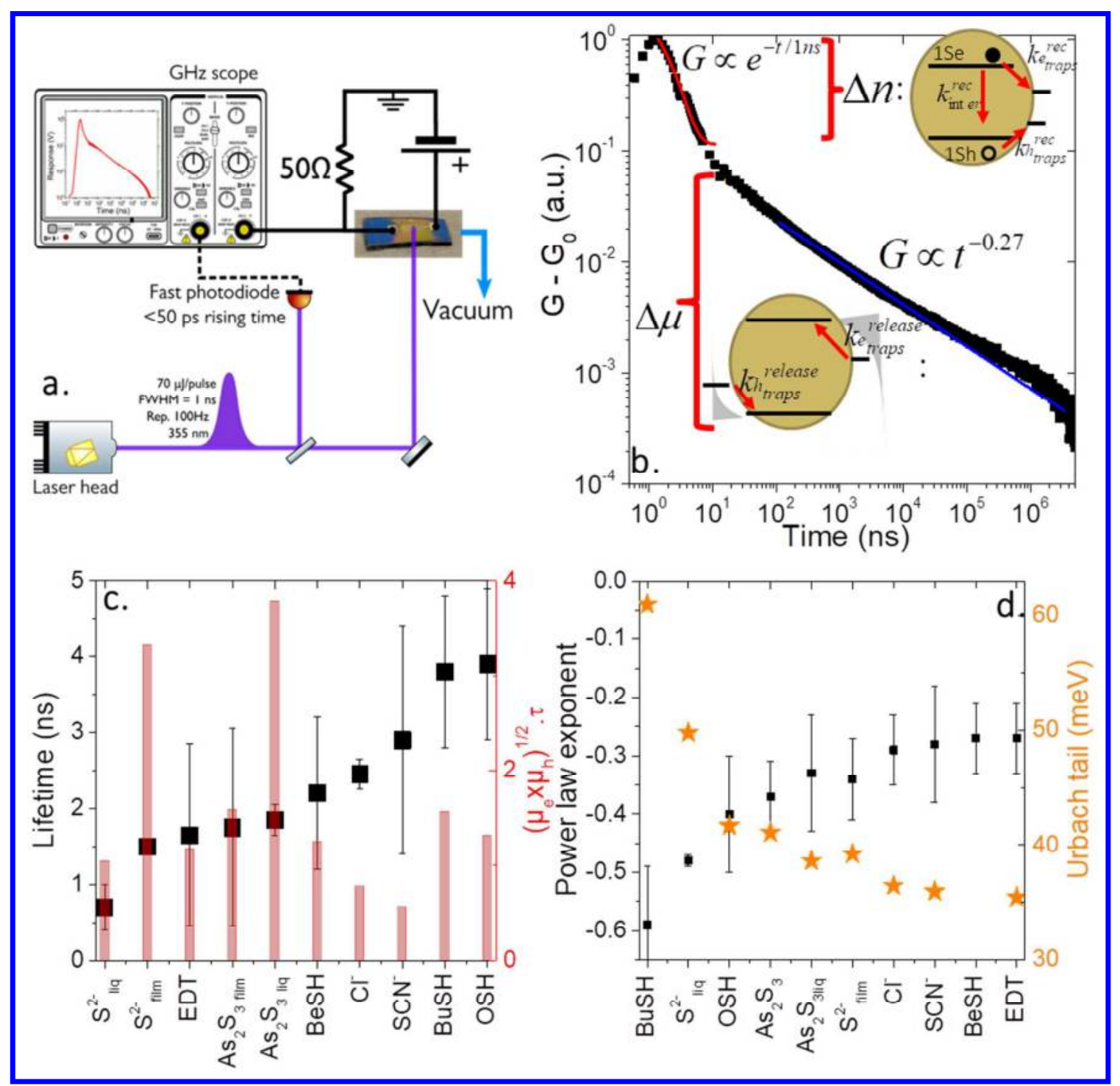

Figure 3a. Scheme of the transient photocurrent setup. b. Typical conductance curve for a film of EDT capped HgTe CQD as a function of time after its illumination by a $1 \mathrm{~ns}$ pulse of light at $355 \mathrm{~nm}$. c. Exciton lifetime, obtained while fitting the short time scale part of the temporal dependence of the conductance, and its product with mobility for HgTe CQD film capped with different ligands. $d$. Value of the exponent obtained while fitting the long time scale part of the temporal dependence of the conductance and the value of the Urbach energy for HgTe CQD film capped with different ligands.

At longer time scale, from $10 \mathrm{~ns}$ up to several ms, the curve follows a power law and no characteristic time scale (in particular no transit time) can be extracted, see Figure $3 \mathrm{~b}$. The exponent value ( $\left.G \propto t^{b}\right)$ relating the conductance to the decay duration is typically between -0.25 and -0.6 depending on the ligands, see Figure $3 \mathrm{~d}$. The power law dependence of the transient photocurrent has been extensively studied in the mid 80's in the context of amorphous and insulating materials ${ }^{35}$. $\mathrm{As}_{2} \mathrm{Se}_{3}$ is probably the most investigated material ${ }^{36,37}$. The presence of this power law dynamics has been interpreted in term of multi trappipg transport. After illumination of the sample, carriers get 
trapped in a tail of the density of states and they present a null mobility until they get thermally re-excited above the mobility band edge. In this regime the number of carriers is constant and the time that they spend on the trapped state makes them behaving as they were continuously decelerated ${ }^{35}$.

Let's assume an exponentially decreasing tail of the density of states for the trap distribution. The characteristic energy decay of this density of states is called Urbach energy $E_{U}^{38}$. It is possible to relate the power law exponent of the time dependence of the conductance to the value of the Urbach energy through the relation: $b=-1+\frac{k_{b} T}{E_{U}}$. As a result, it is possible to determine the density of trap states from this transient photocurrent measurement. It is an important experimental result because other methods to probe the density such as the measurement of the subband-gap absorption using thermal spectroscopy ${ }^{38}$ are difficult to adapt in the infrared range due to the lower dynamics of the spectrometer. We estimate the Urbach energy to range from $35 \mathrm{meV}$ to $60 \mathrm{meV}$. In the case of $\mathrm{S}^{2-}$, we can observe that liquid phase ligand exchange is leading to a higher Urbach energy. A large Urbach energy can be extremely detrimental and it is typically at the origin of the low open circuit voltage in CQD based solar cell ${ }^{39}$. As a result, $\mathrm{S}^{2-}$ ligands which may have look promising from a mobility perspective appear to be actually poor candidates due to the large amount of traps. On the other hand, short dithiol (BeSH and EDT) leads to the lowest amount of traps. Thus in spite of their intermediate mobility lifetime product, they are the one that bring the best trap passivation.
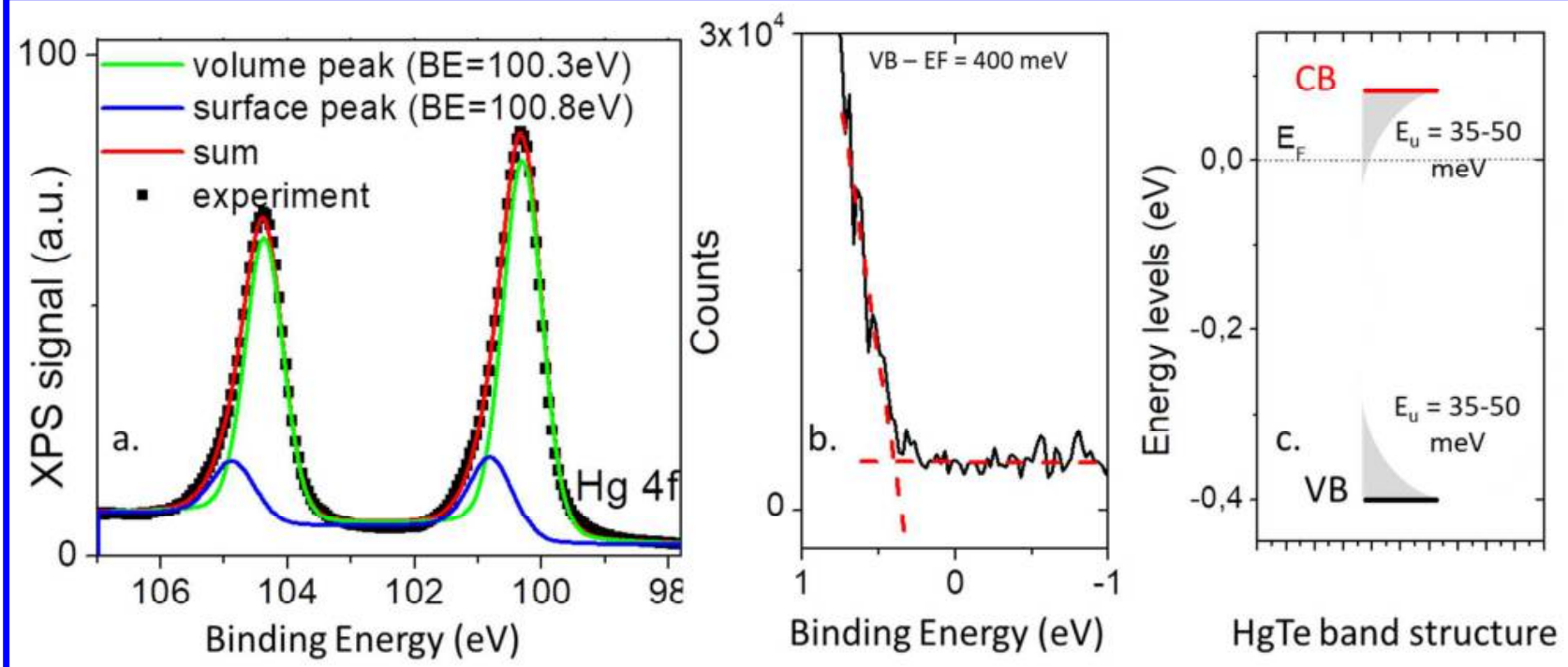

Figure 4 a. X-ray photoemission spectrum of the Hg4f state from $\mathrm{S}^{2-}$ capped HgTe CQD. $b$. X-ray photoemission spectrum of the valence band from $\mathrm{S}^{2-}$ capped HgTe CQD. c. Reconstructed energy spectrum of $\mathrm{S}^{2-}$ capped HgTe CQD. The Fermi level corresponds to 0 eV energy level.

To finish the discussion, we would like to discuss more deeply the exact origin of the trap states. Electron microscopy image (Figure 1b) clearly reveals some size distribution for the CQD. In this case, the large CQD are less confined and may behave as trap states for the small (more confined) CQD. Moreover surface reconstruction and dangling bonds can also generate trap states. To confirm the last hypothesis, we first perform energy-dispersive $\mathrm{X}$-ray measurements which reveal a clear $\mathrm{Hg}$ excess in HgTe nanocrystals, with a $\mathrm{Hg} / \mathrm{Te}$ ratio equal to 1.2 (see Table S1). The excess mercury atoms are localized in surface and get bonded to the ligands which could lead to surface states. Therefore, we use X-Ray photoemission spectroscopy (XPS) and focus on Hg peaks as probe of potential surface states. Measurements have been conducted on the TEMPO beamline of synchrotron SOLEIL. High resolution XPS on the $\mathrm{Hg} 4 \mathrm{f}$ peak reveals the presence of two states, see Figure $4 \mathrm{a}$, while the Te, with no excess in surface, only comes under a single population, see figure $\mathrm{S9}$. The $\mathrm{Hg} 4 \mathrm{f}$ state appears as a doublet with a $4.05 \mathrm{eV}$ spin orbit splitting. After removing the background and fitting the curve with a Voigt shape, we clearly distinguish two contributions. The lowest binding energy (BE) peak has an energy of $100.3 \mathrm{eV}$ and a Full Width at Half Maximum (FWHM) of $0.75 \mathrm{eV}$. 
The second peak presents a $100.8 \mathrm{eV}$ BE with a FWHM of $0.75 \mathrm{eV}$. The ratio of the two peaks can be changed by tuning the photoelectron escape depth. The higher the incident photon energy (ie for large electron escape depth), the smaller the BE peak at higher energies. ${ }^{34}$ As a result we can attribute the second peak to surface states.

Mercury atoms at the surface get bonded to sulfide based ligands, in contrast to bulk $\mathrm{Hg}$ atoms which are bonded to Te. Since sulfur has a higher electronegativity compared to $\mathrm{Te}$, the $\mathrm{Hg}$ atoms coupled to sulfur are less electron rich and this shifts the binding energy toward higher values.

A zoom on the low BE part of the XPS spectrum can be used to extract the location of the valence band with respect to the Fermi level. We obtain a value of $0.40 \mathrm{eV}$, see Figure $4 \mathrm{~b}$. Since the band edge energy is $0.48 \mathrm{eV}$, we conclude that the Fermi level is close to the conduction band (but still in the gap) in this material, this observation being consistent with $(i)$ the transistor measurements which systematically present a negative bias for the charge neutrality point and (ii) recent electrochemical measurements on $\mathrm{n}$-type $\mathrm{HgTe} \operatorname{CQD}^{40}$. Finally we propose a reconstructed energy spectrum for the HgTe CQD which include the trap state distribution, see Figure 4c.

\section{CONCLUSION}

We have investigated the effect of a series of ligands $\left(\mathrm{Cl}^{-}, \mathrm{SCN}^{-}, \mathrm{OSH}, \mathrm{BuSH}, \mathrm{BeSH}, \mathrm{EDT}, \mathrm{As}_{2} \mathrm{~S}_{3}, \mathrm{~S}^{2-}\right)$ on the photoconductive properties of $\mathrm{HgTe}$ with in mind the identification of the capping agent leading to the highest mobility by lifetime product. Surface chemistries based on sulfide ligands seem the more promising. We build a high bandwidth transient photocurrent setup and identify two regimes for the photoconduction. At early time, carriers get recombined and trapped. Then a multiple trapping regime of transport is leading to a power law dependence for the photoconductance. From the fitted exponent we are able to extract the value of the Urbach energy which enables us to evaluate the density of trap states in the gap. In particular, we show that liquid phase exchange is leading to more traps than the solid state approach, which will be quite detrimental for the design of device in a photovoltaic configuration. It appears that the choice of ligand is a tradeoff between performances (ie $\mu \tau$ product) and the amount of induced traps and that short dithiol like EDT and BeSH are around the optimal performance for photoconduction. Finally, we determine by using XPS a full energy spectrum where both quantum and trap states can be located in absolute energy scale.

\section{Supporting Informations}

The Supporting Information is available free of charge on the ACS Publications website at DOI: Preparation of films, optical properties, transport properties in DC and time resolved configuration and photoemission measurements

\section{ACKNOWLEDEGMENTS}

EL thanks the support of ERC starting grant (project: blackQD - grant 756225). We acknowledge the use of clean room facilities from the consortium "Salles Blanches Paris Centre -SBPC". This work has been supported by the Region Ile-de-France in the framework of DIM Nano-K via the grant dopQD. This work was supported by French state funds managed by the ANR within the Investissements d'Avenir program under reference ANR-11-IDEX-0004-02, and more specifically within the framework of the Cluster of Excellence MATISSE.

\section{REFERENCES}

(1) Konstantatos, G.; Sargent, E. H. Colloidal Quantum Dot Optoelectronics and Photovoltaics; Cambridge University Press, 2013. 
(2) Lhuillier, E.; Scarafagio, M.; Hease, P.; Nadal, B.; Aubin, H.; Xu, X. Z.; Lequeux, N.; Patriarche, G.; Ithurria, S.; Dubertret, B. Infrared Photodetection Based on Colloidal Quantum-Dot Films with High Mobility and Optical Absorption up to THz. Nano Lett. 2016, 16, 1282-1286.

(3) Talapin, Dmitri V.; Lee, Jong Soo; Kovalenko, Maksym V.; Shevchenko, Elena V. Prospects of Colloidal Nanocrystals for Electronic and Optoelectronic Applications. Chem Rev 2010, 110, 389458.

(4) Keuleyan, S.; Lhuillier, E.; Brajuskovic, V.; Guyot-Sionnest, P. Mid-Infrared HgTe Colloidal Quantum Dot Photodetectors. Nat. Photonics 2011, 5, 489-493.

(5) Kershaw, S. V.; Susha, A. S.; Rogach, A. L. Narrow Bandgap Colloidal Metal Chalcogenide Quantum Dots: Synthetic Methods, Heterostructures, Assemblies, Electronic and Infrared Optical Properties. Chem. Soc. Rev. 2013, 42, 3033-3087.

(6) Jeong, K. S.; Deng, Z.; Keuleyan, S.; Liu, H.; Guyot-Sionnest, P. Air-Stable N-Doped Colloidal HgS Quantum Dots. J.Phvs. Chem. Lett. 2014, 5, 1139-1143.

(7) Kim, J.; Yoon, B.; Kim, J.; Choi, Y.; Kwon, Y.-W.; Kyu Park, S.; Seob Jeong, K. High Electron Mobility of $\beta$-HgS Colloidal Quantum Dots with Doubly Occupied Quantum States. $\underline{R S C A d v} .2017$, 7, 38166-38170.

(8) Tang, X.; Wu, G. F.; Lai, K. W. C. Plasmon Resonance Enhanced Colloidal HgSe Quantum Dot Filterless Narrowband Photodetectors for Mid-Wave Infrared. JMater Chem C 2017, 5, 362-369.

(9) Robin, A.; Livache, C.; Ithurria, S.; Lacaze, E.; Dubertret, B.; Lhuillier, E. Surface Control of Doping in Self-Doped Nanocrystals. ACS Apol. Mater. Interfaces 2016, 8, 27122-27128.

(10) Jeong, J.; Yoon, B.; Kwon, Y.-W.; Choi, D.; Jeong, K. S. Singly and Doubly Occupied Higher Quantum States in Nanocrystals. Nano Lett. 2017, 17, 1187-1193.

(11) Lhuillier, E.; Keuleyan, S.; Liu, H.; Guyot-Sionnest, P. Mid-IR Colloidal Nanocrystals. Chem. Mater. 2013, 25, 1272-1282.

(12) Kovalenko, M. V.; Kaufmann, E.; Pachinger, D.; Roither, J.; Huber, M.; Stangl, J.; Hesser, G.; Schäffler, F.; Heiss, W. Colloidal HgTe Nanocrystals with Widely Tunable Narrow Band Gap Energies: From Telecommunications to Molecular Vibrations. J.Am. Chem. Soc. 2006, 128, 35163517.

(13) Lhuillier, E.; Keuleyan, S.; Guyot-Sionnest, P. Optical Properties of HgTe Colloidal Quantum Dots. Nanotechnology 2012, 23, 175705.

(14) Chen, M.; Lu, H.; Abdelazim, N. M.; Zhu, Y.; Wang, Z.; Ren, W.; Kershaw, S. V.; Rogach, A. L.; Zhao, N. Mercury Telluride Quantum Dot Based Phototransistor Enabling High-Sensitivity RoomTemperature Photodetection at $2000 \mathrm{Nm}$. ACS Nano 2017, 11, 5614-5622.

(15) Lhuillier, E.; Guyot-Sionnest, P. Recent Progresses in Mid Infrared Nanocrystal Based Optoelectronics. IEEE J. Sel. Top. Quantum Electron. 2017, 23, 1-8.

(16) Guyot-Sionnest, P.; Roberts, J. A. Background Limited Mid-Infrared Photodetection with Photovoltaic HgTe Colloidal Quantum Dots. Appl. Phvs. Lett. 2015, 107, 253104.

(17) Deng, Zhiyou; Jeong, Kwang Seob; Guyot-Sionnest, Philippe. Colloidal Quantum Dots Intraband Photodetectors. ACS Nano 2014, 8 (11), 11707-11714.

(18) Yifat, Y.; Ackerman, M.; Guyot-Sionnest, P. Mid-IR Colloidal Quantum Dot Detectors Enhanced by Optical Nano-Antennas. Anpl.Phvs._Lett. 2017, 110, 41106.

(19) Chen, M.; Shao, L.; Kershaw, S. V.; Yu, H.; Wang, J.; Rogach, A. L.; Zhao, N. Photocurrent Enhancement of HgTe Quantum Dot Photodiodes by Plasmonic Gold Nanorod Structures. ACS Nano 2014, 8, 8208-8216.

(20) Lhuillier, E.; Robin, A.; Ithurria, S.; Aubin, H.; Dubertret, B. Electrolyte-Gated Colloidal Nanoplatelets-Based Phototransistor and Its Use for Bicolor Detection. Nano Lett. 2014, 14, 2715 2719.

(21) Tang, X.; Tang, X.; Lai, K. W. C. Scalable Fabrication of Infrared Detectors with Multispectral Photoresponse Based on Patterned Colloidal Quantum Dot Films. ACS Photonics 2016, 3, 23962404.

(22) Chen, M.; Guyot-Sionnest, P. Reversible Electrochemistry of Mercury Chalcogenide Colloidal Quantum Dot Films. ACS Nano 2017, 11, 4165-4173.

(23) Brown, P. R.; Kim, D.; Lunt, R. R.; Zhao, N.; Bawendi, M. G.; Grossman, J. C.; Bulović, V. Energy Level Modification in Lead Sulfide Quantum Dot Thin Films through Ligand Exchange. ACS Nano 2014, 8, 5863-5872. 
(24) Jeong, K. S.; Tang, J.; Liu, H.; Kim, J.; Schaefer, A. W.; Kemp, K.; Levina, L.; Wang, X.; Hoogland, S.; Debnath, R.; et al. Enhanced Mobility-Lifetime Products in PbS Colloidal Quantum Dot Photovoltaics. ACS Nano 2012, 6, 89-99.

(25) Keuleyan, S.; Lhuillier, E.; Guyot-Sionnest, P. Synthesis of Colloidal HgTe Quantum Dots for Narrow Mid-IR Emission and Detection. J.Am. Chem. Soc. 2011, 133, 16422-16424.

(26) Tang, J.; Kemp, K. W.; Hoogland, S.; Jeong, K. S.; Liu, H.; Levina, L.; Furukawa, M.; Wang, X.; Debnath, R.; Cha, D.; et al. Colloidal-Quantum-Dot Photovoltaics Using Atomic-Ligand Passivation. Nat.Mater. 2011, 10, 765-771.

(27) Fafarman, A. T.; Koh, W.; Diroll, B. T.; Kim, D. K.; Ko, D.-K.; Oh, S. J.; Ye, X.; Doan-Nguyen, V.; Crump, M. R.; Reifsnyder, D. C.; et al. Thiocyanate-Capped Nanocrystal Colloids: Vibrational Reporter of Surface Chemistry and Solution-Based Route to Enhanced Coupling in Nanocrystal Solids. J. Am. Chem. Soc. 2011, 133, 15753-15761.

(28) Lhuillier, E.; Keuleyan, S.; Zolotavin, P.; Guyot-Sionnest, P. Mid-Infrared HgTe/As2S3 Field Effect Transistors and Photodetectors. Adv. Mater. 2013, 25, 137-141.

(29) Nag, A.; Kovalenko, M. V.; Lee, J.-S.; Liu, W.; Spokoyny, B.; Talapin, D. V. Metal-Free Inorganic Ligands for Colloidal Nanocrystals: S2-, HS-, Se2-, HSe-, Te2-, HTe-, TeS32-, OH-, and NH2- as Surface Ligands. J. Am. Chem. Soc. 2011, 133, 10612-10620.

(30) Yakunin, S.; Dirin, D. N.; Protesescu, L.; Sytnyk, M.; Tollabimazraehno, S.; Humer, M.; Hackl, F.; Fromherz, T.; Bodnarchuk, M. I.; Kovalenko, M. V.; et al. High Infrared Photoconductivity in Films of Arsenic-Sulfide-Encapsulated Lead-Sulfide Nanocrystals. ACS Nano 2014, 8, 12883-12894.

(31) Lhuillier, E.; Ithurria, S.; Descamps-Mandine, A.; Douillard, T.; Castaing, R.; Xu, X. Z.; Taberna, P.L.; Simon, P.; Aubin, H.; Dubertret, B. Investigating the N- and P-Type Electrolytic Charging of Colloidal Nanoplatelets. L. Phvs. Chem. C 2015, 119, 21795-21799.

(32) Gao, J.; Fidler, A. F.; Klimov, V. I. Carrier Multiplication Detected through Transient Photocurrent in Device-Grade Films of Lead Selenide Quantum Dots. Nat.Commun. 2015, 6, 8185.

(33) Fidler, A. F.; Gao, J.; Klimov, V. I. Electron-Hole Exchange Blockade and Memory-Less Recombination in Photoexcited Films of Colloidal Quantum Dots. Nat.Phvs. 2017, 13, 604-610.

(34) Martinez, B.; Livache, C.; Notemgnou Mouafo, L. D.; Goubet, N.; Keuleyan, S.; Cruguel, H.; Ithurria, S.; Aubin, H.; Ouerghi, A.; Doudin, B.; et al. HgSe Self-Doped Nanocrystals as a Platform to Investigate the Effects of Vanishing Confinement. ACS Appl. Mater. Interfaces 2017, 9, 3617336180.

(35) Main, C.; Nesheva, D. Transient Photocurrent Techniques as a Means of Characterising Amorphous Semiconductors. J. Optoelectron. Adv. Mater. 2001, 3, 655-664.

(36) Orenstein, J.; Kastner, M. A.; Vaninov, V. Transient Photoconductivity and Photo-Induced Optical Absorption in Amorphous Semiconductors. Philos. Mag. Part B 1982, 46, $23-62$.

(37) Orenstein, J.; Kastner, M. Photocurrent Transient Spectroscopy: Measurement of the Density of Localized States in -As2Se3. Phvs. Rev. Lett. 1981, 46, 1421-1424.

(38) Guyot-Sionnest, P.; Lhuillier, E.; Liu, H. A Mirage Study of CdSe Colloidal Quantum Dot Films, Urbach Tail, and Surface States. J. Chem. Phvs. 2012, 137, 154704.

(39) Guyot-Sionnest, P. Electrical Transport in Colloidal Quantum Dot Films. L.Phvs. Chem. Lett. 2012, 3, 1169-1175.

(40) Shen, G.; Chen, M.; Guyot-Sionnest, P. Synthesis of Nonaggregating HgTe Colloidal Quantum Dots and the Emergence of Air-Stable N-Doping. L.Phvs. Chem.Lett. 2017, 8, 2224-2228. 
TOC graphic

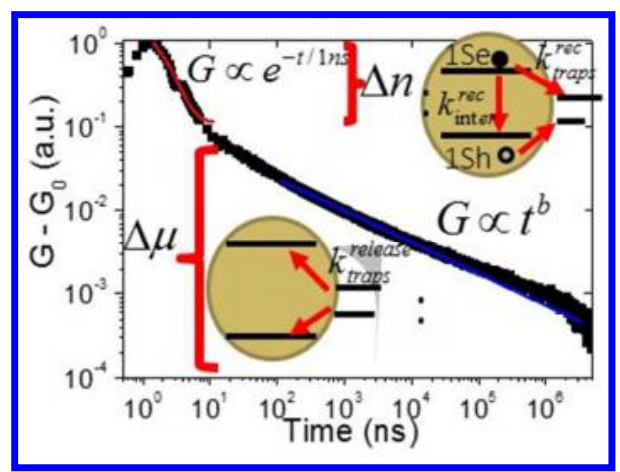

32

33

34

35

36

37

38

39

40

41

42

43

44

45

46

47

48

49

50

53

54

55

56

57

58

59

60

ACS Paragon Plus Environment 